\title{
Histoire globale de la première modernité
}

\section{Sanjay Subrahmanyam}

\section{OpenEdition}

Journals

Édition électronique

URL : https://journals.openedition.org/annuaire-cdf/15964

DOI : 10.4000/annuaire-cdf.15964

ISBN : 978-2-7226-0572-5

ISSN : 2109-9227

Éditeur

Collège de France

Édition imprimée

Date de publication : 30 décembre 2020

Pagination : $575-578$

ISBN : 978-2-7226-0516-9

ISSN : 0069-5580

Référence électronique

Sanjay Subrahmanyam, "Histoire globale de la première modernité », L'annuaire du Collège de France

[En ligne], 118| 2020, mis en ligne le 01 avril 2021, consulté le 22 août 2022. URL : http://

journals.openedition.org/annuaire-cdf/15964 ; DOI : https://doi.org/10.4000/annuaire-cdf.15964 


\title{
HISTOIRE GLOBALE DE LA PREMIÈRE MODERNITÉ
}

\author{
Sanjay SUBRAHMANYAM \\ Professeur à l'université de Californie à Los Angeles, \\ professeur invité au Collège de France
}

Mots-clés : histoire globale, modernité, villes, urbanisme, religion, empires

La série de cours « Réseaux marchands et empires à l'époque moderne » est disponible, en audio et vidéo, sur le site internet du Collège de France (https://www.college-defrance.fr/site/sanjay-subrahmanyam/course-2017-2018.htm) ainsi que le colloque

" Historia insularum : les îles dans l'histoire globale à l'époque moderne " (https://www. college-de-france.fr/site/sanjay-subrahmanyam/symposium-2017-2018.htm).

\section{ENSEIGNEMENT}

\section{COURS - RÉSEAUX MARCHANDS ET EMPIRES À L'ÉPOQUE MODERNE}

Les cours de cette année 2017-2018 étaient consacrés à un sujet considéré classique depuis l'époque de l'enseignement de Fernand Braudel (1902-1985) au Collège de France : la place des réseaux marchands dans les empires de l'époque moderne (c'est-à-dire du XV ${ }^{\mathrm{e}}$ au XVIII ${ }^{\mathrm{e}}$ siècle). Comme on le sait, Braudel a consacré une part importante de son travail à cette question dans le cadre géographique de la Méditerranée chrétienne et musulmane à l'époque moderne, avant d'entamer une réflexion plus large à l'échelle globale dans ses ouvrages portant sur le rapport entre capitalisme et civilisation. Dans la collection de SEVPEN publiée sous sa direction («Ports-Routes-Trafics»), un nombre important d'ouvrages traite des questions liées au commerce des italiens, des marranes, et d'autres groupes encore. Parmi les publications de cette série, il faut également mentionner les collections de lettres marchandes de l'époque médiévale et moderne, qui restent une source importante à la fois pour l'histoire économique et pour l'histoire sociale voire culturelle.

Ces travaux sur les marchands s'inscrivaient dans une histoire longue du capitalisme, débattue par des chercheurs comme Robert S. Lopez, Raymond de Roover, Frederic C. Lane, et Fernand Braudel lui-même. Cependant, l'appareil conceptuel utilisé pour 
analyser ces réseaux a été développé et raffiné plus tard, au début des années 1970, dans le cadre d'une conversation féconde entre historiens et anthropologues. On trouve les débuts de cette conversation dans l'atelier de Karl Polanyi, qui a notamment publié un ouvrage collectif ayant pour titre Commerce et marché dans les premiers empires (édition anglaise : Trade and Market in the Early Empires, 1957). Opposant sa vision d'une « économie encastrée dans le social » à celle d'Adam Smith et sa théorie de la « main invisible », Polanyi a collaboré avec des spécialistes du monde ancien, de l'Inde et de l'Afrique occidentale, pour développer des idées comme le « port-de-commerce », ou de l'opposition complémentaire entre réciprocité, redistribution, et échange. Cependant, Polanyi et ses collègues n'ont pas prêté beaucoup d'attention au rapport entre commerce et ethnicité. C'est au début des années 1970 que l'anthropologue et africaniste Abner Cohen a traité la question dans son essai « Stratégies culturelles dans l'organisation des diasporas commerçantes ${ }^{1}$ ». Cohen a repris et généralisé la notion de diaspora, utilisée jusque-là pour parler essentiellement des communautés juives. Pour Cohen, une diaspora était tout simplement « un groupe ethnique donné ayant la haute main sur la plupart, sinon sur toutes les étapes du négoce de certaines marchandises ». Selon sa définition, « une diaspora commerçante [est] composée de communautés étroitement liées entre elles, quoique dispersées », et en même temps « se distingue des autres groupes sociaux par sa culture et sa structure».

Ce petit texte aurait probablement été oublié en dehors du milieu des africanistes s'il n'avait pas été repris comme point de départ par le grand historien américain Philip D. Curtin dans son ouvrage Cross-Cultural Trade in World History ${ }^{2}$ (1984). Membre-fondateur de l'École de Wisconsin en histoire globale, Curtin a proposé un survol de la place des diasporas commerçantes dans l'histoire du monde. Les premiers chapitres de ce livre traitent du cas de l'Afrique, dont Curtin était spécialiste. Puis, il passe rapidement en revue le monde ancien (Mésopotamie, Assyrie, Égypte, etc.), avant de consacrer deux chapitres à l'histoire des marchands en Chine et en Asie orientale avant 1500. Le cœur du livre est constitué par les chapitres où Curtin développe une analyse de ce qu'il conçoit comme les grandes diasporas de l'époque moderne : Arméniens, Bāniyās indiens, Chinois d'outremer, Ibériques, et compagnies de commerce européennes. L'ouvrage de Curtin, considéré comme fondamental dans les années 1980, a cependant été critiqué par la suite, non seulement à cause de ses nombreuses lacunes, mas aussi pour son usage de l'idée de « diaspora commerçante ». Le grand spécialiste français des réseaux marchands en océan Indien à l'époque contemporaine, Claude Markovits, a proposé par exemple de remplacer le paradigme de « diasporas » par celui de « circulations marchandes ».

Tout en prenant comme point de départ les travaux de Braudel, Polanyi et Curtin, les cours de cette année ont voulu développer un point de vue plus complexe, sur l'interaction entre réseaux marchands et systèmes politiques. Le premier cours a passé en revue quelques grands débats et références essentielles, avant de revenir sur

1. Abner COHEN, «Cultural strategies in the organization of trading diasporas », in C. Meillassoux (dir.), The Development of Indigenous Trade and Markets in West Africa: Studies Presented and Discussed at the Tenth International African Seminar at Fourah Bay College, Freetown, December 1969, Londres, Oxford University Press/International African Institute, 1971, p. 266-278.

2. Philip D. CuRTIN, Cross-Cultural Trade in World History, Cambridge, Cambridge University Press, 1984. 
quelques cas plus particuliers pris dans l'histoire médiévale, notamment de la Genizah du Caire (à l'époque du califat fatimide), étudiée par Shlomo Goitein et ses disciples. Nous avons aussi remarqué le rapport entre l'étude de ces documents et des développements théoriques, établis sur l'emploi de la théorie des jeux et la microéconomie. Le cas de la Genizah est en contraste avec deux autres: des Sogdiens en Asie centrale et en Chine, et les marchands de la Hanse en mer Baltique et ailleurs. Le deuxième cours s'est centré pour l'essentiel sur une étude des marchands italiens dans la longue durée, en commençant avec les réseaux amalfitains $\mathrm{du} \mathrm{VIII}^{\mathrm{e}}$ au XII ${ }^{\mathrm{e}}$ siècle. Cette période a été suivie par une époque lors de laquelle les Génois jouissaient d'une position dominante, parfois partagée avec d'autres villes rivales en Italie. Le rôle de certaines institutions - la commenda, les corporations, et finalement les alberghi (grandes maisons) - dans l'histoire génoise a été souligné. Nous avons également repris la question de la participation des Génois et des Vénitiens dans le commerce asiatique, à partir de l'essor des Mongols comme force politique au XIII ${ }^{\mathrm{e}}$ siècle. Toutefois, des changements importants se sont produits à partir de l'arrivée de la peste noire du XIV e siècle. La dernière partie de ce cours a abordé la rivalité triangulaire entre Génois, Vénitiens et Florentins aux XIVe et $\mathrm{XV}^{\mathrm{e}}$ siècles, avant de terminer sur les réactions différenciées de ces trois villes face à l'expansion ibérique du $\mathrm{XVI}^{\mathrm{e}}$ siècle.

Dans le troisième cours, nous sommes passés de l'Europe à l'Asie, en abordant le cas des Iraniens dans l'océan Indien à l'époque moderne. Le point de départ était les travaux du grand iranisant Jean Aubin, spécialiste de l'histoire de l'Iran avant et après la conquête mongole. Aubin a finement analysé le commerce du golfe Persique (autour du port de Siraf) à l'époque médiévale, et a également produit une série d'essais importants autour du royaume d'Hormuz, centre du commerce entre l'Iran et l'Inde au XV siècle. C'est surtout à partir d'Hormuz que les Iraniens sont rentrés en force dans le monde indien, où ils ont joué un rôle significatif dans le sultanat bahmanide (en Inde occidentale). L'éclatement de ce sultanat à la fin du $\mathrm{XV}^{\mathrm{e}}$ siècle a créé davantage d'opportunités pour les Iraniens. Nous avons développé l'idée d'une double circulation : celle du petit peuple, souvent des spécialistes des arts militaires, et celle des hommes de prestige et des lettrés, comme les grands Sayyids de la mer Caspienne. Parmi les premiers, nous nous sommes penchés sur la carrière de Yusuf Lari (ou As'ad Khan), grand seigneur de guerre dans la région de Belgaum (Karnataka) dans la première moitié du XVI ${ }^{\mathrm{e}}$ siècle, bâtisseur de mosquées, et devenu un homme saint après sa mort. Nous avons également suivi le sort de ces Iraniens dans le Deccan oriental (sultanat de Golconde), avec des cas comme celui de Mir Muhammad Sa'id Ardestani. Pendant le XVII ${ }^{\mathrm{e}}$ siècle, leur réseau a traversé le golfe de Bengale, pour atteindre la Birmanie et la Thaïlande. À la fin du siècle, on trouve la trace de quelques Iraniens jusque dans le Japon des Tokugawas.

Quelques-uns de ces Iraniens trouvent des mentions dans les sources portugaises $\mathrm{du} \mathrm{XVI}^{\mathrm{e}}$ siècle portant sur l'océan Indien, en tant qu'acteurs politiques et commerciaux. Dans le quatrième cours, nous sommes revenus sur la question du commerce dans l'empire portugais, mais cette fois-ci par le biais des réseaux des Juifs et des marranes (nouveaux-chrétiens). Le cours était organisé en trois temps : une première discussion de l'expulsion des Juifs de l'Espagne, leur conversion forcée au Portugal, et la transformation du réseau séfarade en Méditerranée; la participation des nouveaux-chrétiens dans l'empire atlantique portugais, y compris dans le commerce des esclaves entre l'Afrique occidentale et l'Amérique; la question des Juifs séfarades et des marranes en océan Indien, à la fois dans le 
commerce, et dans la circulation des savoirs. Nous avons conclu sur la mission de Menasseh ben Israël en Angleterre à l'époque de Cromwell, et l'intégration des séfarades dans la vie commerciale de l'empire britannique. Les cinquième et sixième cours portaient sur des exemples déjà cités par Curtin : les Chinois d'outremer, et les Arméniens. Dans les deux cas, nous avons profité des avances historiographiques ayant eu cours depuis la publication de son livre en 1984. Dans les deux cas, nous avons pu démontrer que ces réseaux marchands s'impliquaient assez profondément dans la vie politique des sociétés où ils se sont installés (l'Asie du Sud-Est, l'Inde, etc.). L'hypothèse selon laquelle les «diasporas » menaient une vie autonome semble assez affaiblie par l'apport de ces recherches récentes.

\section{COLLOQUE - HISTORIA INSULARUM : LES ÎLES DANS L'HISTOIRE GLOBALE À L'ÉPOQUE MODERNE}

L'importance des îles dans la réalité et l'imaginaire de l'époque moderne est un sujet capital. Il suffit de penser à l'Utopie de Thomas More, ou au cas de Robinson Crusoe, deux des exemples les plus célèbres de cette époque. Dans ce colloque, nous avons réuni des historiens spécialistes des océans et des mers assez divers: la Méditerranée, l'Atlantique, la mer Rouge, l'océan Indien. Chacun a traité le cas d'une île ou d'un archipel, par rapport à une problématique: l'articulation des réseaux de commerce, la colonisation, les ordres militaires et leur fonctionnement, les systèmes sociaux et les conflits entre normes.

- Sanjay Subrahmanyam : «Introduction : les îles dans l'histoire globale »;

- Ingrid Houssaye Michienzi (CNRS) : «Majorque, porte de l'Afrique : le destin d'une île à la croisée des routes $\left(\mathrm{XIV}^{\mathrm{e}}-\mathrm{XV}^{\mathrm{e}}\right.$ siècles) »;

- Roxani Margariti (Emory University) : «For Pearls and Forts: Red Sea islands and the onset of the "Age of Contained Conflict" in the Indian Ocean »;

- Emanuel Buttigieg (L-Università ta’ Malta) : «The Island Order State on Malta: The early modern Maltese archipelago and the Order of St John »;

- Pier M. Larson (The Johns Hopkins University) : «Food, sex and law at Fort Dauphin (Madagascar): France's first colony in the Indian Ocean, 1642-74 »;

- Pernille Røge (University of Pittsburgh) : «From Islands to Empire: The French colonial administration at Gorée and Saint-Louis, 1763-89»;

- Sanjay Subrahmanyam : «Conclusions ».

\section{PuBLicATIONS}

Subrahmanyam S., L'Inde sous les yeux de l'Europe. Mots, peuples, empires (1500-1800), trad. J BlaYAC, Paris, Alma Éditeur, coll. «Essai. Histoire», 2018.

SUbRAHMANYAM S. et AlAM M., «Mediterranean exemplars: Jesuit political lessons for a Mughal emperor », in G. MARCOCCI et L. BIASIORI (dir.), Machiavelli, Islam, and the East: Reorienting the Foundations of Modern Political Thought, Basingstoke, Palgrave Macmillan, 2018, p. 105-29.

SubRAHMANYAM S., « The hidden face of Surat: Reflections on a cosmopolitan Indian Ocean centre, 1540-1750 », Journal of the Economic and Social History of the Orient, vol. 61, $\mathrm{n}^{\text {os }} 1-2,2018$, p. 205-255, DOI : 10.1163/15685209-12341448.

SUBRAHMANYAM S., «Semper per viam portugalensem. Some Neglected Aspects of Italians in Sixteenth-Century Portuguese Asia », Purusārtha, vol. 35 : L'Inde et l'Italie. Rencontres intellectuelles, politiques et artistiques, 2018, p. 25-51. 